\title{
ON THE ABSOLUTE SUMMABILITY OF THE CONJUGATE SERIES OF A FOURIER SERIES
}

\section{T. PATI}

1.1. Let $f(t)$ be a periodic function with period $2 \pi$ and integrable $(L)$ over $(-\pi, \pi)$. Let

$$
\frac{1}{2} a_{0}+\sum_{n=1}^{\infty}\left(a_{n} \cos n t+b_{n} \sin n t\right)
$$

be the Fourier series of $f(t)$. Then the conjugate series, or the allied series, of the Fourier series (1.1.1) is given by

$$
\sum_{n=1}^{\infty}\left(b_{n} \cos n t-a_{n} \sin n t\right) \text {. }
$$

Let

$$
\psi(t)=\frac{1}{2}\{f(x+t)-f(x-t)\}, \quad \theta(t)=\frac{2}{\pi} \int_{t}^{\infty} \frac{\psi(u)}{u} d u .
$$

Suppose throughout $t>0$. We write

$$
\begin{aligned}
& \Psi_{\alpha}(t)=\frac{1}{\Gamma(\alpha)} \int_{0}^{t}(t-u)^{\alpha-1} \psi(u) d u, \quad \alpha>0, \\
& \Psi_{0}(t)=\psi(t),
\end{aligned}
$$

and

$$
\psi_{\alpha}(t)=\Gamma(\alpha+1) t^{-\alpha} \Psi_{\alpha}(t), \quad \alpha \geqq 0 .
$$

Similarly

$$
\begin{aligned}
& \Theta_{\alpha}(t)=\frac{1}{\Gamma(\alpha)} \int_{0}^{t}(t-u)^{\alpha-1} \theta(u) d u, \quad \alpha>0, \\
& \Theta_{0}(t)=\theta(t)
\end{aligned}
$$

and

$$
\theta_{\alpha}(t)=\Gamma(\alpha+1) t^{-\alpha} \Theta_{\alpha}(t), \quad \alpha \geqq 0 .
$$

1.2. The object of this paper is to study the nature of the conjugate series regarding its absolute summability.

In their paper On the absolute summability of the allied series of $a$

Received by the editors August 27, 1951 and, in revised form, March 10, 1952. 
Fourier series [2] Bosanquet and Hyslop established the following:

TheOREM A. If $\alpha \geqq 0$, and $\psi_{\alpha}(t)$ is of bounded variation in $(0, \pi)$ and $\theta_{\lambda}(t)$ is of bounded variation in $(0, \pi)$ for some positive $\lambda$, then the allied series is summable $|C, \alpha+\delta|$ for every $\delta>0$.

By making use of an example they also proved that when $\alpha=0$, this theorem is the best of its kind, that is to say, the theorem will fail when $\delta=0$. In $\$ 2.1$ I prove that also when $\alpha=1$, the theorem will continue to remain the best of its kind.

1.3. It was shown by Whittaker [9] with the help of an example, suggested by Littlewood, that a Fourier series may converge at a point without being summable $|A|$ at that point, while Prasad [6] constructed an example of a series which is summable $|A|$ at a point without being convergent at that point. This shows that the properties of convergence and summability $|A|$ of infinite series are independent of each other. Fekete [4] proved that while summability $|C|$ implies summability $|A|$, the converse is not necessarily true. A natural question would be to examine the summability $|C|$ of Fourier series and conjugate series, when the series are not only summable $|A|$, but also convergent.

In $\$ 3.1$ I show for Fourier series and conjugate series that summability $|A|$, even when coupled with convergence, does not necessarily imply summability $|C, 1|$ of these series.

My grateful thanks are due to Dr. B. N. Prasad for his kind help and encouragement during the preparation of this paper.

2.1. In this section I establish the following:

THEOREM 1. If $\psi_{1}(t)$ is of bounded variation in $(0, \pi)$ and $\theta_{\lambda}(t)$ is of bounded variation in $(0, \pi)$ for some positive $\lambda$, then the conjugate series, although summable $|C, 1+\delta|$ for every $\delta>0$, is not necessarily summable $|C, 1|$.

2.2. I shall first prove that Theorem $\mathrm{A}$ of $\$ 1.2$, for $\alpha=1$, is equivalent to the following theorem of Bosanquet and Hyslop [2], for $\beta=0$.

THEOREM B. If $\beta \geqq 0$, and if

$$
\int_{0}^{\eta} \frac{\left|\psi_{\beta}(t)\right|}{t} d t<\infty,
$$

where $\eta>0$, then the allied series is summable $|C, \beta+1+\delta|$ at the point $t=x$, for every $\delta>0$.

I shall then take up an example for which the integral $\left(^{*}\right)$ for $\beta=0$ exists, but the corresponding conjugate series is not summable $|C, 1|$. 
2.3. In order to prove that Theorem $\mathrm{A}$ for $\alpha=1$ is equivalent to Theorem $\mathrm{B}$ for $\beta=0$, we require the following lemma.

LEммA. If $\alpha \geqq 0$, then the necessary and sufficient conditions that $\theta_{\alpha}(t)$ should be of bounded variation in $(0, \eta)$, where $\eta>0$, are that $\psi_{\alpha+1}(t)$ and $\theta_{\lambda}(t)$ should be of bounded variation in $(0, \eta)$ for some positive $\lambda$.

For the proof of the lemma, reference may be made to Lemma 7 of the paper of Bosanquet and Hyslop.

In virtue of the lemma, when $\alpha \geqq 1$, the hypotheses in Theorem $A$ are the necessary and sufficient conditions that $\theta_{\alpha-1}(t)$ should be of bounded variation in $(0, \pi)$. Now, since $\theta_{\alpha-1}(t)$ is an integral for $t>0$, it is a function of bounded variation in every interval $(\eta, \pi), 0<\eta<\pi$. Hence, the hypotheses in Theorem $A$ are equivalent to the bounded variation of $\theta_{\alpha-1}(t)$ in $(0, \eta)$, that is, to the convergence of the integral

$$
\int_{0}^{\eta} \frac{\left|\psi_{\alpha-1}(t)\right|}{t} d t
$$

since, by a result due to Bosanquet [1], for $\alpha \geqq 1,(1 / 2) \pi \theta_{\alpha-1}^{\prime}(t)$ $=-\psi_{\alpha-1}(t) / t$, except possibly at a set of points of measure zero. The equivalence referred to in $\$ 2.2$ follows immediately by taking $\alpha=1$.

2.4. Now let us consider the series

$$
-\sum_{n=2}^{\infty} \frac{\sin n \alpha}{\log n},
$$

which is the conjugate series of the Fourier series

$$
\sum_{n=2}^{\infty} \frac{\cos n \alpha}{\log n}
$$

it being known (Titchmarsh $[8, \S 13.51]$ ) that $(2.4 .1)$ is not itself a Fourier series.

The series (2.4.1) is convergent for all values of $\alpha$. For $\alpha=\pi / 2$, however, it is not absolutely convergent. Using the result (Kogbetliantz [5]) that if $\sum_{1}^{\infty} a_{n}$ is summable $|C, 1|$, then $\sum_{1}^{\infty}\left|a_{n}\right| / n$ is convergent, we see that (2.4.1) is not even summable $|C, 1|$ at $\alpha=\pi / 2$.

Now, if $f(\alpha)$ be the sum-function of (2.4.2) for $0<\alpha<2 \pi$, that is, the generating function of (2.4.2), then (Young [10])

$$
\begin{aligned}
f(\alpha)= & -\frac{1}{2} \cos \alpha-(\pi-\alpha) \sin \alpha \\
& +\frac{\pi}{2} \int_{0}^{\infty} \frac{\cosh y(\pi-\alpha)}{\sinh \pi y\left\{\pi^{2} / 4+(\log y)^{2}\right\}} d y .
\end{aligned}
$$


Therefore, for $0 \leqq t<\pi / 2$,

$$
\begin{aligned}
\psi(t) & =\frac{1}{2}\left\{f\left(\frac{\pi}{2}+t\right)-f\left(\frac{\pi}{2}-t\right)\right\} \\
& =\frac{1}{2} \sin t+t \cos t-\frac{\pi}{4} \int_{0}^{\infty} \frac{\sinh y t}{\cosh \frac{y \pi}{2}\left\{\frac{\pi^{2}}{4}+(\log y)^{2}\right\}} d y .
\end{aligned}
$$

Hence,

$$
|\psi(t)| \leqq \frac{1}{2} \sin t+t \cos t+\frac{\pi}{4} \int_{0}^{\infty} \frac{\sinh y t}{\cosh \frac{y \pi}{2}\left\{\frac{\pi^{2}}{4}+(\log y)^{2}\right\}} d y .
$$

Let $0<\eta<\pi / 2$. Then

$$
\begin{aligned}
\int_{0}^{\eta} \frac{|\psi(t)|}{t} d t \leqq & \frac{1}{2} \int_{0}^{\eta} \frac{\sin t}{t} d t+\int_{0}^{\eta} \cos t d t \\
& +\frac{\pi}{4} \int_{0}^{\eta} \frac{d t}{t} \int_{0}^{\infty} \frac{\sinh y t}{\cosh \frac{y \pi}{2}\left\{\frac{\pi^{2}}{4}+(\log y)^{2}\right\}} d y .
\end{aligned}
$$

Now,

$$
\begin{aligned}
I & \equiv \int_{0}^{\eta} \frac{d t}{t} \int_{0}^{\infty} \frac{\sinh y t}{\cosh \frac{y \pi}{2}\left\{\frac{\pi^{2}}{4}+(\log y)^{2}\right\}} d y \\
& =\int_{0}^{\infty} \frac{d y}{\cosh \frac{y \pi}{2}\left\{\frac{\pi^{2}}{4}+(\log y)^{2}\right\}} \int_{0}^{\frac{\sinh y t}{t}} d t
\end{aligned}
$$

if we change the order of integration by Fubini's theorem. Since $(\sinh y t) / t \leqq y \cosh y t$, we have $\int_{0}^{\eta}((\sinh y t) / t) d t \leqq \sinh y \eta$. This could also be inferred from term-by-term integration. Therefore,

$$
\begin{aligned}
I & \leqq \int_{0}^{\infty} \frac{\sinh y \eta}{\cosh \frac{y \pi}{2}\left\{\frac{\pi^{2}}{4}+(\log y)^{2}\right\}} d y \\
& =\frac{2}{\pi}\left\{\sin \eta+2 \eta \cos \eta+f\left(\frac{\pi}{2}-\eta\right)-f\left(\frac{\pi}{2}+\eta\right)\right\},
\end{aligned}
$$


which is finite for every $\eta$, such that $0<\eta<\pi / 2$. Also, since

$$
\int_{0}^{\eta} \frac{\sin t}{t} d t<\infty
$$

we finally have, for $0<\eta<\pi / 2$,

$$
\int_{0}^{\eta} \frac{|\psi(t)|}{t} d t<\infty .
$$

Thus, in the case of the conjugate series (2.4.1), at $\alpha=\pi / 2$,

$$
\int_{0}^{\eta} \frac{\left|\psi_{0}(t)\right|}{t} d t<\infty,
$$

and still the series is not summable $|C, 1|$.

Combining the statements of $\$ \S 2.2,2.3$, and 2.4 , we obtain the theorem of $\$ 2.1$.

3.1. For the case of the Fourier series, it can be demonstrated, as explained below, that summability $|A|$, coupled with convergence, does not necessarily imply summability $|C, 1|$.

Bosanquet and Kestelman [3] established that summability $|C, 1|$ of a Fourier series is not a local property. Thus, the bounded variation of $f(t)$ in the immediate neighbourhood of the point considered, $t=x$, is not sufficient to ensure the summability $|C, 1|$ of the corresponding Fourier series. On the other hand, we know from Jordan's criterion for the convergence of a Fourier series and from a theorem of Prasad [7] for the summability $|A|$ of Fourier series that bounded variation of $f(t)$ in the immediate neighbourhood of the point $t=x$ is sufficient to ensure the convergence and summability $|A|$, respectively, of the Fourier series of $f(t)$ at $t=x$. Thus, it is possible for a Fourier series to be both summable $|A|$ and convergent at a point without being necessarily summable $|C, 1|$ at that point.

For proving that even for conjugate series summability $|A|$, together with convergence, does not ensure summability $|C, 1|$ of the series, we shall make use of the example already mentioned in \$2.4. The series (2.4.1) is summable $|A|$ at $\alpha=\pi / 2$, since by a theorem of Prasad [7] the convergence of the integral

$$
\int_{0}^{\eta} \frac{|\psi(t)|}{t} d t
$$

where $\eta>0$, is a sufficient condition for summability $|A|$. The series (2.4.1) is also convergent for all values of $\alpha$. Yet, as has already been shown, it is not summable $|C, 1|$ at $\alpha=\pi / 2$. 
3.2. Thus, with the help of the example of the series (2.4.1), we obtain the following theorem.

THEOREM 2. For the conjugate series of a Fourier series summability $|A|$ at a point, even when coupled with everywhere-convergence, does not necessarily imply summability $|C, 1|$ at that point.

\section{REFERENCES}

1. L. S. Bosanquet, On the Cesdro summation of Fourier series and allied series, Proc. London Math. Soc. (2) vol. 37 (1934) pp. 17-32.

2. L. S. Bosanquet and J. M. Hyslop, On the absolute summability of the allied series of a Fourier series, Math. Zeit. vol. 42 (1937) pp. 489-512.

3. L. S. Bosanquet and H. Kestelman, The absolute convergence of a series of integrals, Proc. London Math. Soc. (2) vol. 45 (1939) pp. 88-97.

4. M. Fekete, On the absolute summability $(A)$ of infinite series, Proceedings of the Edinburgh Mathematical Society (2) vol. 3 (1932) pp. 132-134.

5. E. Kogbetliantz, Sur les séries absolument sommables par la méthode des moyennes arithmétiques, Bull. Sci. Math. (2) vol. 49 (1925) pp. 234-256.

6. B. N. Prasad, The absolute summability $(A)$ of Fourier series, Proceedings of the Edinburgh Mathematical Society (2) vol. 2 (1931) pp. 129-134.

7. - On the summability of Fourier series and the bounded variation of power series, Proc. London Math. Soc. (2) vol. 35 (1933) pp. 407-424.

8. E. C. Titchmarsh, The theory of functions, Oxford, 1939.

9. J. M. Whittaker, The absolute summability of Fourier series, Proceedings of the Edinburgh Mathematical Society (2) vol. 2 (1930) pp. 1-5.

10. W. H. Young, On the Fourier series of bounded functions, Proc. London Math. Soc. (2) vol. 12 (1913) pp. 41-70.

University of Allahabad 